\title{
Characteristic Ratio and Its Properties
}

\author{
Lijuan Chen (Corresponding author) \& Mingzhu Li \\ School of Science, Qingdao Technological University \\ Fushun Road 11, Qingdao, China \\ E-mail: chenljcool@163.com
}

\begin{abstract}
Similar to the definition of cross ratio in high geometry, we propose a new definition of characteristic ratio. This paper mainly discuss some properties of characteristic ratio. Moreover, we find this definition plays an important role in researching the intrinsic of algebraic curve in projective plane.
\end{abstract}

Keywords: Characteristic ratio, Projective plane, Projective coordinate

\section{Introduction}

As well known, cross ratio is the basic invariant in projective geometry, which is defined by collinear four points. It can explain the projective mapping of two planes. In paper [Luo, 2008], the authors find an equivalence relation between the study of the intrinsic properties of plane algebraic curves and the study of singularity of bivariate spline spaces when studying computational geometry. From that, they proposed the definitions of characteristic ratio and characteristic mapping.

First, we list the definition of characteristic ratio and characteristic mapping. In what follows, we have used $u=<a, b>$ for the intersection point of lines $a$ and $b, a=(u, v)$ for the line determined by the points $u$ and $v$.

Definition 1(Characteristic Ratio) Let $u, v \in P^{2}$ be two points(or lines), $l_{1}, l_{2}, \cdots, l_{k}$ be distinct points(or lines) on the line determined by $u, v$ and $l_{i}=a_{i} u+b_{i} v, i=1,2, \cdots, k$. The ratio

$$
\left[u, v ; l_{1}, l_{2}, \cdots, l_{k}\right]=\frac{b_{1} b_{2} \cdots b_{k}}{a_{1} a_{2} \cdots a_{k}}
$$

is called characteristic ratio of $l_{1}, l_{2}, \cdots . l_{k}$ to the basic points (or basic lines ) $u, v$.

Especially, in projective plane, suppose $P_{1}, P_{2}, P_{3}, P_{4}$ are four different points in a line, and $P_{3}=\lambda_{1} P_{1}+\lambda_{2} P_{2}, P_{4}=$ $\mu_{1} P_{1}+\mu_{2} P_{2}$. Denoted

$$
\left[P_{1}, P_{2} ; P_{3}, P_{4}\right]=\frac{\mu_{2} \lambda_{2}}{\mu_{1} \lambda_{1}},
$$

then $\left[P_{1}, P_{2} ; P_{3}, P_{4}\right]$ is the characteristic ratio of basic points $P_{1}, P_{2}$.

Theorem 2 In projective plane, suppose $P_{1}, P_{2}, P_{3}, P_{4}$ are four different points, and $P_{i}=P+\lambda_{i} Q(i=1,2,3,4)$, where $P, Q$ are two defferent points in this line, then

$$
\left[P_{1}, P_{2} ; P_{3}, P_{4}\right]=\frac{\left(\lambda_{1}-\lambda_{3}\right)\left(\lambda_{1}-\lambda_{4}\right)}{\left(\lambda_{2}-\lambda_{3}\right)\left(\lambda_{2}-\lambda_{4}\right)} .
$$

Proof: Let $P+\lambda_{3} Q, P+\lambda_{4} Q$ indicate the linear combination of $P+\lambda_{1} Q, P+\lambda_{2} Q$. The result can be proven from the definition of characteristic ratio.

In Theorem 2, the characteristic ratio of points $P_{1}, P_{2}, P_{3}, P_{4}$ has nothing to the selection of $P, Q$. In fact, if choose points $P^{\prime}$ and $Q^{\prime}$ in this line and suppose $P_{i}=P^{\prime}+\lambda_{i}^{\prime} Q^{\prime}(i=1,2,3,4)$, then we can prove

$$
\frac{\left(\lambda_{1}-\lambda_{3}\right)\left(\lambda_{1}-\lambda_{4}\right)}{\left(\lambda_{2}-\lambda_{3}\right)\left(\lambda_{2}-\lambda_{4}\right)}=\frac{\left(\lambda_{1}^{\prime}-\lambda_{3}^{\prime}\right)\left(\lambda_{1}^{\prime}-\lambda_{4}^{\prime}\right)}{\left(\lambda_{2}^{\prime}-\lambda_{3}^{\prime}\right)\left(\lambda_{2}^{\prime}-\lambda_{4}^{\prime}\right)} .
$$

Definition 3 (Characteristic mapping) 
Let $P_{1}, P_{2}, P_{3}, P_{4} \in P^{2}$ be collinear points, the mapping $\chi_{\left(P_{1}, P_{2}\right)}: P_{3} \longmapsto P_{4}$ is called a characteristic mapping if $\left[P_{1}, P_{2} ; P_{3}, P_{4}\right]=1$ holds, and the characteristic is denoted by $P_{4}=\chi_{\left(P_{1}, P_{2}\right)}\left(P_{3}\right)$.

It can be seen that if $P_{3}$ is the characteristic mapping of $P_{4}$, then $P_{4}$ is is the characteristic mapping of $P_{3}$ as well. That is, the characteristic mapping is reflexive mapping. i.e. $\chi_{\left(P_{1}, P_{2}\right)} \cdot \chi_{\left(P_{1}, P_{2}\right)}=I$ (Identity mapping).

\section{Properties of characteristic ratio}

Theorem 4 In projective plane, the characteristic ratio of four different points in a line remain unchanged under center projective.

Proof In projective plane, suppose $A, B, C, D$ in line $l$ turn into $A^{\prime}, B^{\prime}, C^{\prime}, D^{\prime}$ in line $l^{\prime}$ under center projective $S$ respectively(See Figure 1). Without loss generality, we regard coordinate system $\left[A^{\prime}, B^{\prime}, A ; B\right]$, and let $A^{\prime}(1,0,0), B^{\prime}(0,1,0), A(0,0,1), B(1,1$ We have $l_{A A^{\prime}}=(0,1,0), l_{B B^{\prime}}=(1,0,-1), S=A A^{\prime} \times B B^{\prime}=(1,0,1)$, where $A A^{\prime} \times B B^{\prime}$ denotes the points of intersection of the lines $A A^{\prime}$ and $B B^{\prime}$.

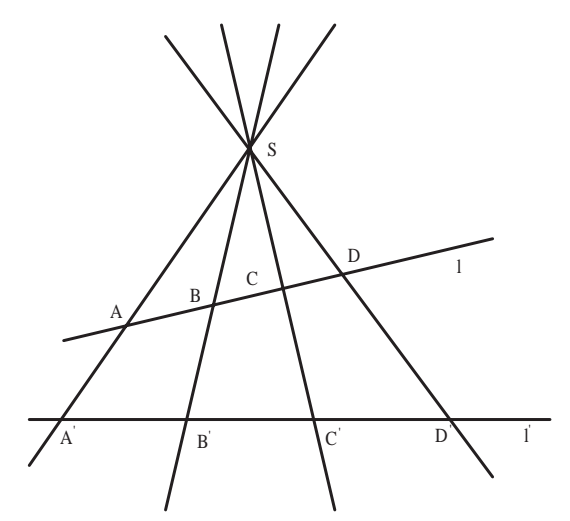

Figure 1 Center Projective

Suppose

$$
\begin{aligned}
& C=A+\lambda B=\left(\begin{array}{l}
0 \\
0 \\
1
\end{array}\right)+\lambda\left(\begin{array}{l}
1 \\
1 \\
1
\end{array}\right)=\left(\begin{array}{c}
\lambda \\
\lambda \\
1+\lambda
\end{array}\right), \\
& D=A+\mu B=\left(\begin{array}{l}
0 \\
0 \\
1
\end{array}\right)+\mu\left(\begin{array}{l}
1 \\
1 \\
1
\end{array}\right)=\left(\begin{array}{c}
\mu \\
\mu \\
1+\mu
\end{array}\right),
\end{aligned}
$$

then $[A, B ; C, D]=\lambda \mu$.

Further, suppose

$$
\begin{aligned}
& C^{\prime}=A^{\prime}+\lambda^{\prime} B^{\prime}=\left(\begin{array}{l}
1 \\
0 \\
0
\end{array}\right)+\lambda^{\prime}\left(\begin{array}{l}
0 \\
1 \\
0
\end{array}\right)=\left(\begin{array}{l}
1 \\
\lambda^{\prime} \\
0
\end{array}\right), \\
& D^{\prime}=A^{\prime}+\mu^{\prime} B^{\prime}=\left(\begin{array}{c}
1 \\
\mu^{\prime} \\
0,
\end{array}\right),
\end{aligned}
$$

Since

$$
C^{\prime}=C+k S=\left(\begin{array}{c}
\lambda \\
\lambda \\
1+\lambda
\end{array}\right)+k\left(\begin{array}{l}
1 \\
0 \\
1
\end{array}\right)=\left(\begin{array}{c}
\lambda+k \\
\lambda \\
1+\lambda+k
\end{array}\right)
$$

The direct result by comparing (2) and (3) is

$$
\left\{\begin{array}{l}
\lambda+k=\rho \\
\lambda=\rho \lambda^{\prime}(\rho \neq 0), \\
1+\lambda+k=0
\end{array}\right.
$$

So $\lambda^{\prime}=-\lambda$. Similarly $\mu^{\prime}=-\mu$.

Therefore $[A, B ; C, D]=\lambda \mu=\left(-\lambda^{\prime}\right)\left(-\mu^{\prime}\right)=\left[A^{\prime}, B^{\prime} ; C^{\prime}, D^{\prime}\right]$. 
Theorem 5 In projective plane, suppose $P_{1}, P_{2}, P_{3}, P_{4}$ are four different points in a line, then the properties of their characteristic ratio are as follows:

(1) Exchange two basic points, the characteristic ratio is reciprocal of the original one, i.e. $\left[P_{2}, P_{1} ; P_{3}, P_{4}\right]=\frac{1}{\left[P_{1}, P_{2} ; P_{3}, P_{4}\right]}$;

(2) Exchange two distinguished points, the characteristic ratio is unchanged, i.e. $\left[P_{1}, P_{2} ; P_{4}, P_{3}\right]=\left[P_{1}, P_{2} ; P_{3}, P_{4}\right]$;

(3) Exchange two basic points and two distinguished points simultaneously, the characteristic ratio is reciprocal of the original one, i.e. $\left[P_{2}, P_{1} ; P_{4}, P_{3}\right]=\frac{1}{\left[P_{1}, P_{2} ; P_{3}, P_{4}\right]}$.

Proof:

(1) Suppose $P_{3}=\lambda_{1} P_{1}+\lambda_{2} P_{2}, P_{4}=\mu_{1} P_{1}+\mu_{2} P_{2}$,

then

$$
\left[P_{1}, P_{2} ; P_{3}, P_{4}\right]=\frac{\lambda_{2} \mu_{2}}{\lambda_{1} \mu_{1}}=\alpha,\left[P_{2}, P_{1} ; P_{3}, P_{4}\right]=\frac{\lambda_{1} \mu_{1}}{\lambda_{2} \mu_{2}}=\frac{1}{\alpha} .
$$

(2) Suppose $P_{3}=\lambda_{1} P_{1}+\lambda_{2} P_{2}, P_{4}=\mu_{1} P_{1}+\mu_{2} P_{2}$,

then

$$
\left[P_{1}, P_{2} ; P_{3}, P_{4}\right]=\frac{\lambda_{2} \mu_{2}}{\lambda_{1} \mu_{1}}=\alpha,\left[P_{2}, P_{1} ; P_{3}, P_{4}\right]=\frac{\lambda_{2} \mu_{2}}{\lambda_{1} \mu_{1}}=\alpha
$$

(3) From (1) and (2), it can be easily proven.

Theorem 6 Suppose three points of the four different points in a line and their characteristic ratio are known, the fourth point can be determined.

\section{Application of Characteristic Ratio in Algebraic Curves}

The following two theorem explain the importance of characteristic ratio in algebraic curve.

Theorem 7 Suppose $P, Q, R \in \mathbf{P}^{2}$ be three different points, if $P, Q, R$ are collinear, then

$$
[P, Q ; R] \cdot[Q, R ; P] \cdot[R, P ; Q]=1 .
$$

Proof: Since $P, Q, R$ are collinear, without loss of generality, suppose $P=a Q+b R$, So

$$
Q=-\frac{b}{a} R+\frac{1}{a} P, R=\frac{1}{b} P-\frac{a}{b} Q .
$$

and

$$
[P, Q ; R] \cdot[Q, R ; P] \cdot[R, P ; Q]=\frac{-\frac{a}{b}}{\frac{1}{b}} \cdot \frac{b}{a} \cdot \frac{\frac{1}{a}}{-\frac{b}{a}}=1 .
$$

From Definition 3 and Theorem 7, we have

Corollary $\mathbf{8}$ Soppose $P, Q, R \in \mathbf{P}^{2}$ are three different points, if $P, Q, R$ are collinear, then

$$
\left[P, Q ; \chi_{(P, Q)}(R)\right] \cdot\left[Q, R ; \chi_{(Q, R)}(P)\right] \cdot\left[R, P ; \chi_{(R, P)}(Q)\right]=1
$$

Theorem 9 Let $P_{1}, P_{2}, \cdots, P_{6} \in \mathbf{P}^{2}$ be any distinct points and $a=\left(P_{1}, P_{2}\right), b=\left(P_{3}, P_{4}\right), c=\left(P_{5}, P_{6}\right)$. Denoted by $u=\langle a, c\rangle, v=\langle b, c\rangle, w=\left\langle a, b>\right.$, then $P_{1}, P_{2}, \cdots, P_{6}$ lie on a conic curve if and only if

$$
\left[u, w ; P_{1}, P_{2}\right] \cdot\left[w, v ; P_{3}, P_{4}\right] \cdot\left[v, u ; P_{5}, P_{6}\right]=1 .
$$

Proof: Without loss of generality, suppose that $u=(1,0,0), v=(0,1,0), w=(0,0,1)$, and then

$$
a=w \times u=(0,1,0), b=v \times w=(0,0,1), c=u \times v=(1,0,0)
$$

Let

$$
\begin{aligned}
& P_{1}=\left(a_{1}, 0, b_{1}\right), P_{2}=\left(a_{2}, 0, b_{2}\right), P_{3}=\left(0, a_{3}, b_{3}\right), \\
& P_{4}=\left(0, a_{4}, b_{4}\right), P_{5}=\left(a_{5}, b_{5}, 0\right), P_{6}=\left(a_{6}, b_{6}, 0\right) .
\end{aligned}
$$

By Pascal Theorem, the equivalent condition that $P_{1}, P_{2}, \cdots, P_{6}$ lie on a conic is that

$$
\left(<\left(P_{1}, P_{5}\right),\left(P_{2}, P_{6}\right)>,<\left(P_{3}, P_{6}\right),\left(P_{1}, P_{4}\right)>,<\left(P_{2}, P_{4}\right),\left(P_{3}, P_{5}\right)>\right)=0 .
$$


By computation,

$$
\begin{aligned}
& \left(<\left(P_{1}, P_{5}\right),\left(P_{2}, P_{6}\right)>,<\left(P_{3}, P_{6}\right),\left(P_{1}, P_{4}\right)>,<\left(P_{2}, P_{4}\right),\left(P_{3}, P_{5}\right)>\right) \\
& =\left(a_{6} b_{5}-a_{5} b_{6}\right)\left(a_{1} b_{2}-a_{2} b_{1}\right)\left(a_{3} b_{4}-a_{4} b_{3}\right)\left(b_{1} b_{2} b_{3} b_{4} b_{5} b_{6}-a_{1} a_{2} a_{3} a_{4} a_{5} a_{6}\right) .
\end{aligned}
$$

Since $P_{1}, P_{2}, P_{3}, P_{4}, P_{5}, P_{6}$ is different,

$$
\left(a_{6} b_{5}-a_{5} b_{6}\right)\left(a_{1} b_{2}-a_{2} b_{1}\right)\left(a_{3} b_{4}-a_{4} b_{3}\right) \neq 0 .
$$

(4) is equivalent that

$$
\frac{b_{1} b_{2} b_{3} b_{4} b_{5} b_{6}}{a_{1} a_{2} a_{3} a_{4} a_{5} a_{6}}=1
$$

Also

$$
\left[u, w ; P_{1}, P_{2}\right] \cdot\left[w, v ; P_{3}, P_{4}\right] \cdot\left[v, u ; P_{5}, P_{6}\right]=\frac{b_{1} b_{2} b_{3} b_{4} b_{5} b_{6}}{a_{1} a_{2} a_{3} a_{4} a_{5} a_{6}} .
$$

From (5), this theorem is proven.

It is easily to verify from the above definition 3 and Theorem 9 that

Corollary 10 Let $P_{1}, P_{2}, \cdots, P_{6} \in \mathbf{P}^{2}$ be any distinct points and $a=\left(P_{1}, P_{2}\right), b=\left(P_{3}, P_{4}\right), c=\left(P_{5}, P_{6}\right)$. Denoted by $u=\langle a, c\rangle, v=\langle b, c\rangle, w=<a, b>$, then $P_{1}, P_{2}, \cdots, P_{6}$ lie on a conic curve if and only if

$$
\left[u, w ; \chi_{(u, w)}\left(P_{1}\right), \chi_{(u, w)}\left(P_{2}\right)\right] \cdot\left[w, v ; \chi_{(w, v)}\left(P_{3}\right), \chi_{(w, v)}\left(P_{4}\right)\right] \cdot\left[v, u ; \chi_{(v, u)}\left(P_{5}\right), \chi_{(v, u)}\left(P_{6}\right)\right]=1 .
$$

Corollary 11 Let $P_{1}, P_{2}, \cdots, P_{6} \in \mathbf{P}^{2}$ be any distinct points and $a=\left(P_{1}, P_{2}\right), b=\left(P_{3}, P_{4}\right), c=\left(P_{5}, P_{6}\right)$. Denoted by $u=\langle a, c\rangle, v=\langle b, c\rangle, w=\left\langle a, b>\right.$, then $\chi_{(u, w)}\left(P_{1}\right), \chi_{(u, w)}\left(P_{2}\right), \chi_{(w, v)}\left(P_{3}\right), \chi_{(w, v)}\left(P_{4}\right), \chi_{(v, u)}\left(P_{5}\right), \chi_{(v, u)}\left(P_{6}\right)$ lie on a conic curve if and only if

$$
\left[u, w ; P_{1}, P_{2}\right] \cdot\left[w, v ; P_{3}, P_{4}\right] \cdot\left[v, u ; P_{5}, P_{6}\right]=1 .
$$

\section{Conclusion}

In this paper, we discuss the properties of characteristic ratio and find that the characteristic ratio plays an important role in intrinsic property of algebraic curve. Those conclusion can help us to study the corresponding relationship about point and line in projective plane. Also, the intrinsic property of algebraic curve. Moreover, we should further study the property of characteristic mapping, which can help us to research computational mathematics and high geometry.

\section{References}

Luo, Zhongxuan, Chen, Lijuan \& Liu, Yu. Intrinsic properties and invariant of algebraic curves in the algebraic plane, submitted to computational geometry.

Luo, Zhongxuan \& Chen, Lijuan. (2005). The singularity of $S_{\mu+1}^{\mu}\left(\triangle_{M S}^{\mu}\right)$, Journal of information and computational science, 2(4):739-746.

Mei, Xiangpeng \& Liu, Zengxian. (2000). High Geometry. high education press, Beijing.

Zhu, Dexiang \& Zhu, Weizong. (2007). High Geometry. High education press, Beijing. 